\title{
Signal Jump Detection Process
}

\author{
Rohit Chandrashekhar Iyer \\ Department of Electronics \\ Engineering \\ Priyadarshini College of \\ Engineering Nagpur, \\ Maharashtra, India
}

\author{
Rohit S. Garode \\ Department of Electronics \\ Engineering \\ Priyadarshini College of \\ Engineering Nagpur, \\ Maharashtra, India
}

\author{
Aniket P. Bhoyar \\ Department of Electronics \\ Engineering \\ Priyadarshini College of \\ Engineering Nagpur, \\ Maharashtra, India
}

\begin{abstract}
Now a days we see that various types of accidents happens on the road. In India many accidents are caused due to human negligence. License Plate detection and recognition is a key technique in most of the traffic related applications such as signal jumping road traffic monitoring, airport gate monitoring, speed monitoring and Automatic parking access control .It is simply the ability to automatically extract and recognition of the vehicle license number plate's character from a captured image .In this paper, we try to give an enhance view of the signal jump detection and recognition of number plate.

Keywords: Signal jump detection, Number plate detection, Plate Recognition, Image processing, ANPR system, Vehicles detection, character detection
\end{abstract}

\section{INTRODUCTION}

With the growth of the urbanization, industrialization and population, there has been a tremendous growth in the traffic. There is occurrence of bundle of problems too, these problems include signal jump, traffic jams, accidents and traffic rule violation. In 1868, the traffic lights only installed in London and today these have installed in every cities around the world. Today red light violation is one of the most common and serious problem which results in the collision of millions of vehicles at the traffic light signals every year. A red light violation occurs when a vehicle try to cross the intersection at the red traffic light. So to give the punishment to the drivers of these vehicles, we must identify the vehicle that violates the traffic light signals

Number Plate Recognition (NPR) is an image technology used to identify plates for their vehicles. This technology is gaining popularity in security and traffic facilities. The purpose of NPR was to build a system capable of automatically recording of the license plate numbers of signal jump traveling down a roadway.

\section{EASE OF USE}

\subsection{Use of Microcontroller ATmega16[5]}

Standard for a microcontroller based low cost platform. It consists of an Atmel ATmega16 is a low power CMOS 8-bit microcontroller based on the AVR enhanced RISC architecture. By executing powerful instructions in a single clock cycle, the ATmega16 achieves throughputs approaching 1 MIPS per MHz allowing the system designed to optimize power consumption versus processing speed. The AVR core combines a rich instruction set with 32 general purpose working registers. All the 32 registers are directly connected to the Arithmetic Logic Unit (ALU), allowing two independent registers to be accessed in one single instruction executed in one clock cycle. The resulting architecture is more code efficient while achieving throughputs up to ten times faster than conventional CISC microcontrollers.

The ATmega16 provides the following features: $16 \mathrm{~K}$ bytes of In-System Programmable Flash Program memory with Read While Write capabilities, 512 bytes EEPROM, $1 \mathrm{~K}$ byte SRAM, 32 general purpose I/O lines, 32 general purpose working registers, On-chip Debugging support and programming, three flexible Timer/Counters with compare modes, Internal and External Interrupts, a serial programmable USART, a byte oriented Two-wire Serial Interface, an 8-channel, 10-bit ADC with optional differential input stage with programmable gain, a programmable Watchdog Timer with Internal Oscillator, an SPI serial port, and six software selectable power saving modes. The Idle mode stops the CPU while allowing the USART, Two wire interface, A/D Converter, SRAM, Timer/Counters, SPI port, and interrupt system to continue functioning, The ADC Noise Reduction mode stops the CPU and all I/O modules except Asynchronous Timer and ADC, to minimize switching noise during ADC conversions.

1. Atmega 16 run at $16 \mathrm{Mhz}$ clock.8051 run at lower clock speed.

2. Atmega16 has inbuilt ADC.8051 doesn't has ADC.

3. RAM and ROM memory of Atmega16 is more than 8051 .

4. Form Factor of Atmega16 is cheaper than the 8051.

5. Programmer for Atmega16 is cheaper than the 8051.

6. Atmega16 have $16 \mathrm{~kb}$ flash memory and Atmega have 32 $\mathrm{kb}$.

7. Atmega have $1 \mathrm{~kb}$ SRAM and Atmega have 2kb SRAM.

8. Atmega have 512bytes EEPROM and Atmega have $1 \mathrm{~kb}$.

In this project we doesn't need more memory and RAM so we are not using Atmega32. And the cost of Atmega32 is also high.

\subsection{Use of IC-MAX 232(Level shifter)}

MAX 232 converts signals from a RS 232serial port to signals suitable for use in TTL-compatible digital logic circuits. The MAX232 is a dual transmitter / dual receiver that typically is used to convert the RX, TX, CTS, RTS signals. The drivers provide TIA-232 voltage level outputs about \pm 7.5 TO 12 Volts from a single 5-volt supply by on-chip charge pump and external capacitors. When a MAX232 IC receives a TTL level to convert, it changes a TTL logic 0 to between +3 and $+15 \mathrm{~V}$, 
and changes TTL logic 1 to between -3 and $-15 \mathrm{~V}$, and vice versa.

\subsection{Use of IR Sensor}

IR stands for infrared sensor.IR Sensors work by using a specific light sensor to detect a select light wavelength in the Infra-Red (IR) spectrum. By using an LED which produces light at the same wavelength as what the sensor is looking for, you can look at the intensity of the received light. When an object is close to the sensor, the light from the LED bounces off the object and into the light sensor. This results in a large jump in the intensity, which we already know can be detected using a threshold. A electronic remote device mainly consists of this IR transmitter and receiver. The IR signal is modulated during transmission. And demodulator during reception

\subsection{Use of GSM module}

The GSM module has the SIM900A microcontroller, a SIM slot, a $12 \mathrm{~V}$ input, RS232 (Serial) interface and Pin outs for interfacing with controllers. It is suitable for SMS, Voice as well as DATA transfer application in M2M interface.

\section{Block Diagram of Signal Jump Detection Process}

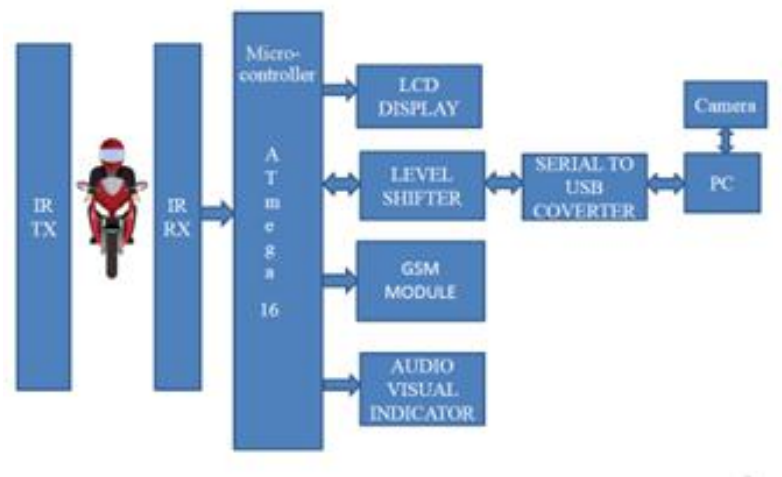

\section{Working}

IR Transmitter and Receiver are connected at line of sight on the two sides of the road. If the vehicle is between Transmitter and Receiver there is deflection in output of IR receiver and this signal is given to microcontroller. After the sensing is done, the signal will be received by the Atmega 16 microcontroller which is used in interfacing the sensors and the computer which is used to find the number of the vehicle. The audio and visual indicator is there to give help to the traffic inspector on the signal that someone has broken the signal. As the signal is broken by the vehicle the Atmega 16 will start working and the
LCD display connected is there to display that from where side the signal is broken. At the output of Atmega16 there is level shifter connected to it. The level shifter is mainly connected to administer the appropriate voltage to the computer (Laptop) as it runs on $12 \mathrm{v}$ and the output of the Atmega16 is approx. 5-6v. And if the level shifter is not connected there will be garbage value or unwarranted results will show up. The tool which we are using for NPR (Number Plate Recognition) is MATLAB12.0. The flowchart of processes in MATLAB is as follows.

\section{PC, MATLAB AND CAMERA TYPESET TEXT}

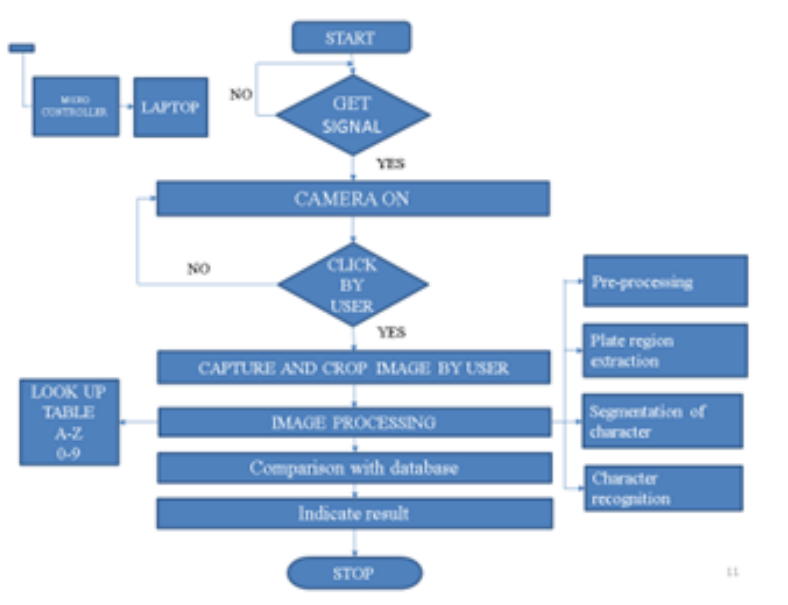

The main and the most important portion of this system is the software model. The software model uses series of image processing techniques which are implemented using MATLAB. The NPR algorithm is broadly divided into following parts.

1.Capture image.

2. Pre-processing.

3. Plate region extraction.

4. Segmentation of character.

5. Character recognition.

6. Comparison with database.

7. Indicate result.

\subsection{Capture Image}

The first step is the capturing of an image using camera. For this project, the test images of vehicles are taken with a camera which is there on the Laptop. The images will be stored as colour JPEG format in system. The next step is to use the Matlab function to convert the captured vehicle JPEG image into gray scale format. 


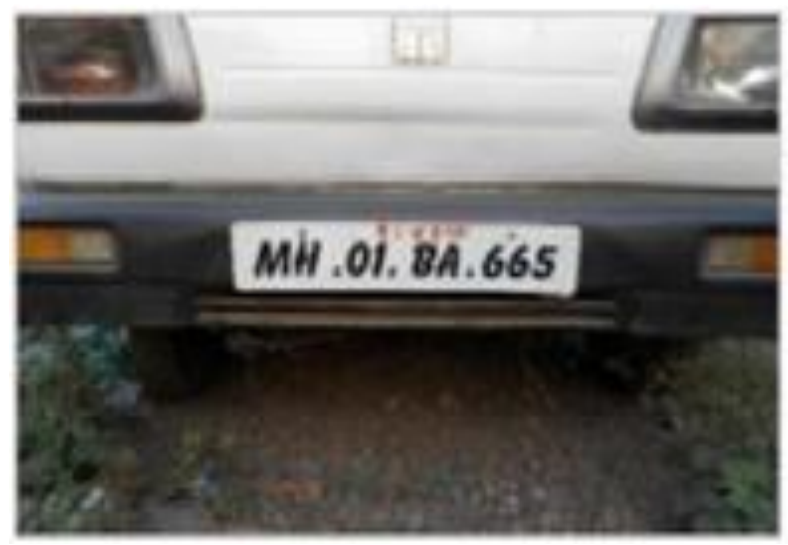

Figure 1 Original Captured Image

\subsection{Pre-processing}

Filtering: When the image is saved, there is noise present in the image. To remove noise from the image median filters are used so that image becomes free from noise. Noise removal is necessary step in License plate recognition system because it greatly affects the recognition rate of the system. Gray Processing: It involves conversion of color image into a gray image. The method is based on different color transform. According to the R, G, B value in the image, it calculates the value of gray value, and obtains the gray image at the same time.

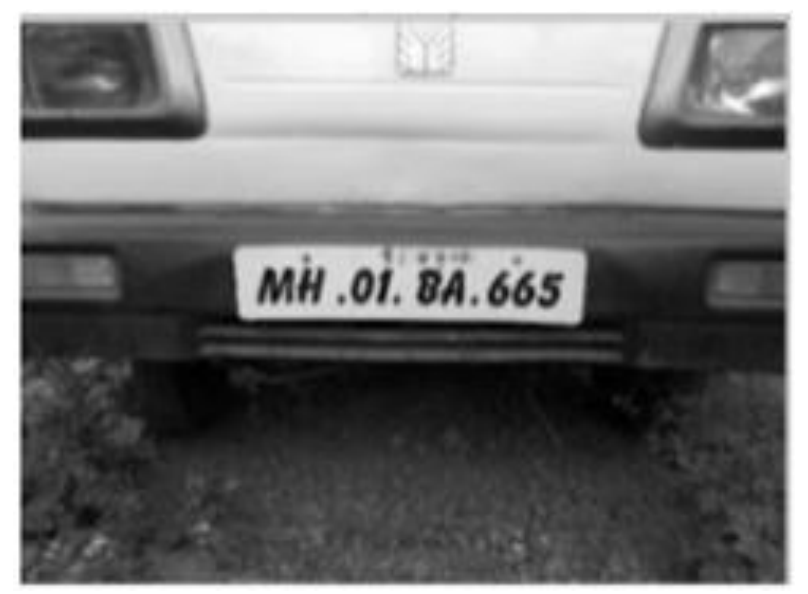

Figure 2 Grey Scale image

\subsection{Plate Region Extraction[2]}

The most important step in the process of automatic number plate recognition is a detection of a number plate area. We can include algorithms that are able to detect a rectangular area of the number plate in an original image. Human beings define a number plate in a natural language as a "small plastic or metal plate attached to a vehicle for official identification purposes", but machines do not understand this definition as well as they do not understand what "vehicle", "road", or whatever else is. Because of this, there is a need to find an alternative definition of a number plate based on descriptors that will be comprehensible for machines. Let us define the number plate as a "rectangular area with increased occurrence of horizontal and vertical edges". The high density of horizontal and vertical edges on a small area is in many cases caused by contrast characters of a number plate, but not in every case. This process can sometimes detect a wrong area that does not correspond to a number plate. Because of this, we often detect several candidates for the plate by this algorithm, and then we choose the best one by a further heuristic analysis. The edges for an image are always the important characteristics that offer an indication for a higher frequency. Detection of edges for an image may help for image segmentation, data compression, and also help for well matching, such as image reconstruction and so on.

There are many methods to make edge detection. The most common method for edge detection is to calculate the differentiation of an image. The first-order derivatives in an image are computed using the gradient, and the second order derivatives are obtained using the Laplacian. Another method for edge detection uses Hilbert Transform. A number plate can be extracted by using image segmentation method. There are numerous image segmentation methods available in various literatures. In most of the methods image binarization is used. To find the region of image we will calculate the centroid and boundary and we have some condition we will apply the further procedure as shown to extract the number plate in MATLAB. Following figure shows the extracted number plate.

\section{Equations:-}

$\mathrm{b}=\mathrm{a}(\mathrm{R} / 3: \mathrm{R}, 1: \mathrm{C})$;

$\mathrm{a}=$ Original Image, $\mathrm{R}=$ Row, $\mathrm{C}=$ column .

Find the area number using following equations

$\mathrm{B}=$ STATS. BoundingBox;

$\mathrm{Xmin}=\mathrm{B}(2)$;

$\mathrm{X} \max =\mathrm{B}(2)+\mathrm{B}(4)$

$\mathrm{Ymin}=\mathrm{B}(1)$;

$\mathrm{Ymax}=\mathrm{B}(1)+\mathrm{B}(3)$

LP=b $(X \min +25: X \max -20, Y \min +10: Y \max -10)$;

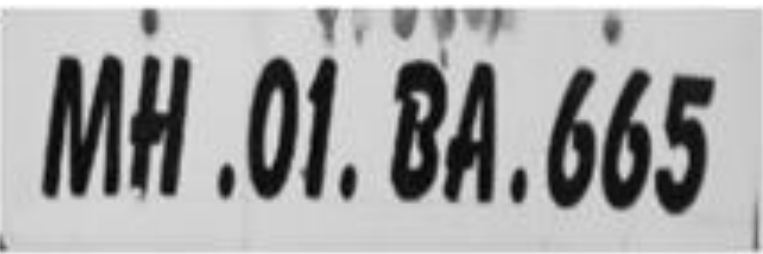

Figure 3 Cropped Image Number Plate 


\section{MH .01. BA. 665}

Figure 4 Filtered Extracted Number Plate

\subsection{Segmentation of Character[1]}

The next step after the detection of the number plate area is a segmentation of the plate. The segmentation is one of the most important processes in the automatic number plate recognition, because all further steps rely on it. If the segmentation fails, a character can be improperly divided into two pieces, or two characters can be improperly merged together. We can use a horizontal projection of a number plate for the segmentation, or one of the more sophisticated methods, such as segmentation using the neural networks. If we assume only one-row plates, the segmentation is a process of finding horizontal boundaries between characters. The second phase of the segmentation is an enhancement of segments. The segment of a plate contains besides the character also undesirable elements such as dots and stretches as well as redundant space on the sides of character. There is a need to eliminate these elements and extract only the character.

Segmentation of plate using a horizontal projection. Since the segmented plate is deskewed, we can segment it by detecting spaces in its horizontal projection. We often apply the adaptive thresholding filter to enhance an area of the plate before segmentation. The adaptive thresholding is used to separate dark foreground from light background with nonuniform illumination.

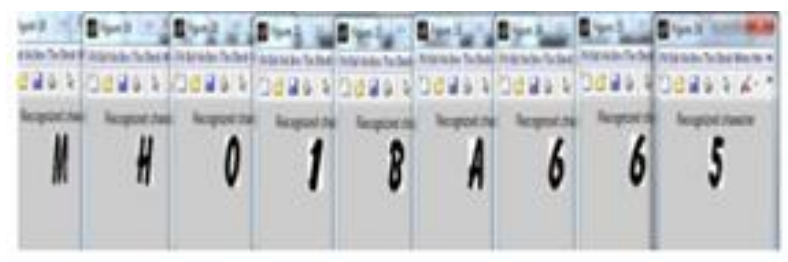

Figure 5 Character Segmentation

\subsection{Look up table[3]}

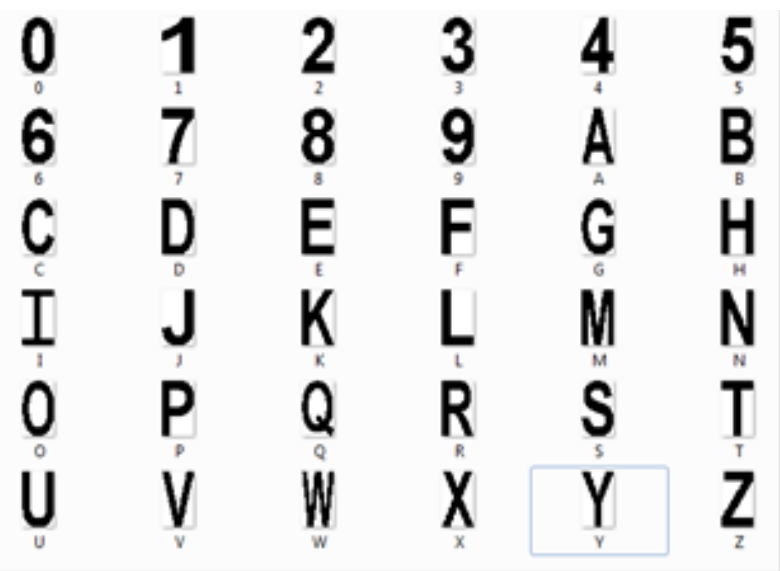

\subsection{Character recognition and Comparison with look up table[3]}

The segmented character is now used to compare with individual character against the complete alphanumeric look up table. It match individual character and finally the number is identified and stored in string format in a variable. The string is then compared with the stored database for the vehicle authorization then recognized number plate string is compare with authenticated database file, , if the both value is same means it will display the authorized otherwise it will display the unauthorized.

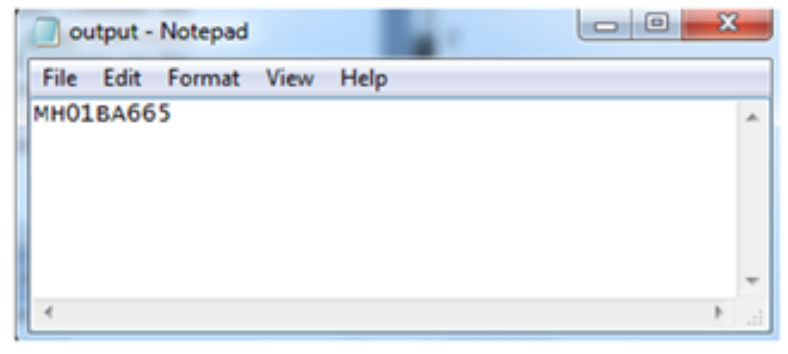

Figure 6 Recognized Number Plate

\section{Conclusion}

In this system, an application software is designed for the detection of number plate of vehicles using their number plate. At first plate location is extracted using edge detection operation then separated the plate characters individually by segmentation. Finally template matching is applied with the use of correlation for recognition of plate characters. In this project we are trying to reduce traffic violation in India which is the biggest problem facing in India. And take strict action on them. Some of possible difficulties:

Broken number plate.

Blurry images.

Number plate not within the legal specification.

Low resolution of the characters.

Poor maintenance of the vehicle plate. 


\section{REFERENCES}

[1] Chetan Sharma and Amandeep Kaur "Indian Vehicle License Plate Extraction and Segmentation" International Journal of Computer Science and Communication Vol. No. 2, pp. 593-599, JulyDecember 2011

[2] Kumar Parasuraman, Member, IEEE and P.Vasantha Kumar, "An Efficient Method for Indian Vehicle License Plate Extraction and Character Segmentation", 2010 IEEE International Conference on Computational Intelligence and Computing Research.

[3] P.Subbuthai, Azha Perisamy and S.Muruganand, "Identifying the Character by Applying
PCAMethod using Matlab" International Journal of Computer Applications (0975-8887), Volume 60No.1, December 2012.

[4] Chitode. J. S, Rupali Kate, "Number Plate Recognition Using Segmentation", International Journal of Engineering Research \& Tehnology (IJERT), Vol. 1 Issue 9, and ISSN: 2278-0181, 2012.

[5] Microcontroller Datasheet At Mega 16, www.atmel.com/devices/atmega 16

[6] Chirag N. Paunwala, Suprava Patnaik, "A Novel Multiple License Plate Extraction Technique for Complex Background in Indian Traffic Conditions", In Proceedings of International Journal of Image Processing, vol.4, issue2, 2007 